\title{
Arteriovenous fistula of superficial temporal vessels
}

\author{
E. Biegaj', J. Rutkowska-Zimirska', M. Radzymińska-Maliszewska ${ }^{1}$, A. Zaremba², J. Pniewski ${ }^{1}$ \\ ${ }^{1}$ Department of Neurology, Czerniakowski Hospital, Warsaw, Poland \\ 2Department of General and Oncological Surgery, Mazovian Specialist Hospital, Radom, Poland
}

[Received: 4 December 2018; Accepted: 14 January 2019]

\begin{abstract}
We present a case of patient with a rare vessel pathology — arteriovenous fistula (AVF) of superficial temporal artery and vein. The 56-year-old man was admitted to the Department of Neurology because of a headache in the right temporal region with concomitant buzzing sound in his right ear. The pain was present mainly in the evenings and was stronger when touching the temporal region. He denied having any head injury in the last few years. There were no signs of central nervous system involvement in the neurological examination. Within the right temporal area a subcutaneous mass with redness of the surrounding skin and with palpable and audible pulsatile thrill was observed. In computed tomography (CT) of the head no abnormalities were found. In duplex-Doppler ultrasound examination of carotid arteries the systolic blood velocity in the right external carotid artery was over two times higher than in the left one. Its flow profile was turbulent and low-resistant. In CT angiography (CTA) an AVF between superficial temporal artery and vein was revealed - it was located at the level of the right zygomatic arch. In both CTA and magnetic resonance angiography, no abnormal connections between extra- and intracranial vessels were found. The patient underwent surgery with good result - all the symptoms disappeared. AVFs of vessels of the scalp are rare and their aetiology is mainly traumatic or iatrogenic. By describing this case we wanted to draw attention to less frequent causes of headache. (Folia Morphol 2019; 78, 4: 879-882)
\end{abstract}

Key words: arteriovenous malformation, superficial temporal artery, headache, vascular surgery

\section{INTRODUCTION}

According to the International Classification of Headache Disorders ( $3^{\text {rd }}$ Edition), headaches are divided into the following categories: primary headaches, secondary headaches and painful cranial neuropathies, other facial pains, and other headaches. Secondary headaches are often defined as symptoms of vascular pathologies within the head and neck. These include dural arteriovenous fistulas (AVFs) and unruptured arteriovenous malformations (AVM). The following case study leads us to believe that headaches can be caused by specifically localised AVMs.

\section{CASE REPORT}

A 56-year-old white male was admitted to the Department of Neurology because of a headache lasting for approximately 1 month. The pain was localised in the right temporal area and was mostly induced by laying on the right side as well as on palpation, and while combing the hair. The patient emphasized that the pain was more intense towards the end of the day. In addition, the patient complained of a concomitant pulsatile tinnitus or buzzing sound in the right ear, which he described as "a heartbeat". Patient denied taking any prescription or over-the-counter medica-

Address for correspondence: Dr. E. Biegaj, Department of Neurology, Czerniakowski Hospital, ul. Stępińska 19/25, 00-739 Warszawa, Poland, e-mail: biegaj.ewa85@gmail.com 


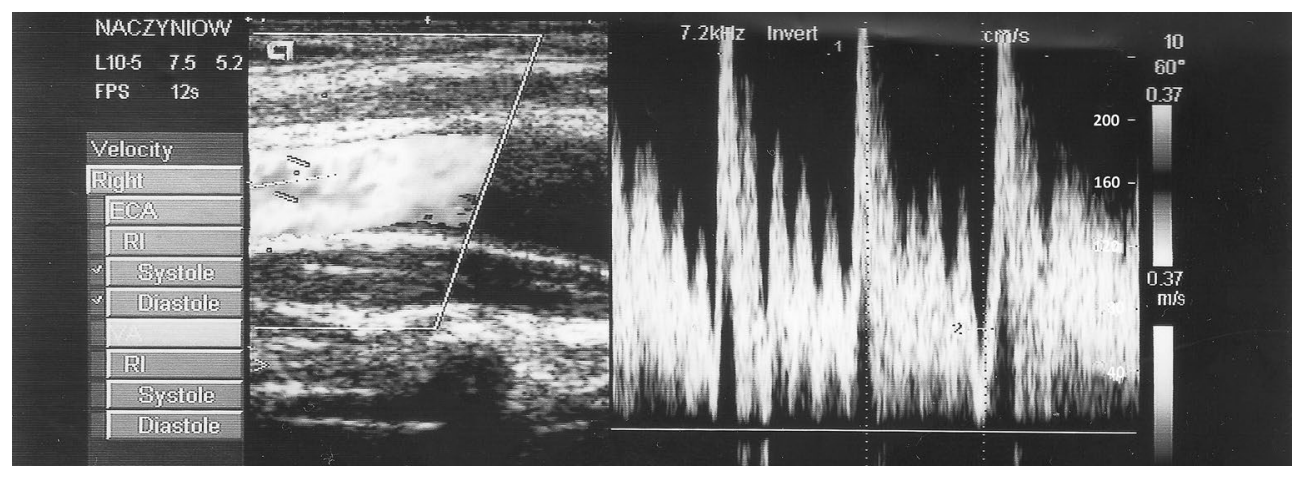

Figure 1. Abnormal accelerated, low resistant flow in the right external carotid artery.

tion at the time. He has a history of 20 plus years of smoking cigarettes and alcohol abuse in the past. A couple of years earlier he had an unspecified accident that led to injury of the left brachial plexus. Patient was not complaining of any medical consequences of the injury mentioned above. He denied any head injury in the past; there was no loss of consciousness at any time.

Physical examination of the scalp revealed a subcutaneous, non-movable mass, surrounded by a margin of red skin. The hair in the area was sparser than on the opposite side. The mass was pulsatile. There was a bruit heard upon auscultation of the mass. Compression of the proximal segment of the superficial temporal artery caused the disappearance of the pulsation and bruit on the affected side. This symptom is called Terrier's sign and is specific to AVMs. Besides, there was a discrete peripheral paresis of the left upper limb. The rest of the neurological exam was unremarkable.

Provided history and physical exam led us to pursue the suspicion of AVM of the scalp vasculature. Laboratory tests of blood serum did not reveal any abnormalities. Computed tomography (CT; Philips Brilliance 64) of the brain as well as magnetic resonance imaging (MRI; GE Signa 1.5 T) of the brain were unremarkable for any intracranial abnormality. Duplex-Doppler ultrasound of the carotid arteries was performed (Acuson CV70). Apart from small atherosclerotic changes in proximal segments of both the internal carotid arteries, there was an asymmetrical flow profile in the external carotid arteries: normal in the left artery, and low-resistant with high systolic and end-diastolic velocities in the right one (Fig. 1). This anomaly was strongly suggestive of AVF on the right side. Magnetic resonance angiography (MRA) revealed widened, elon-

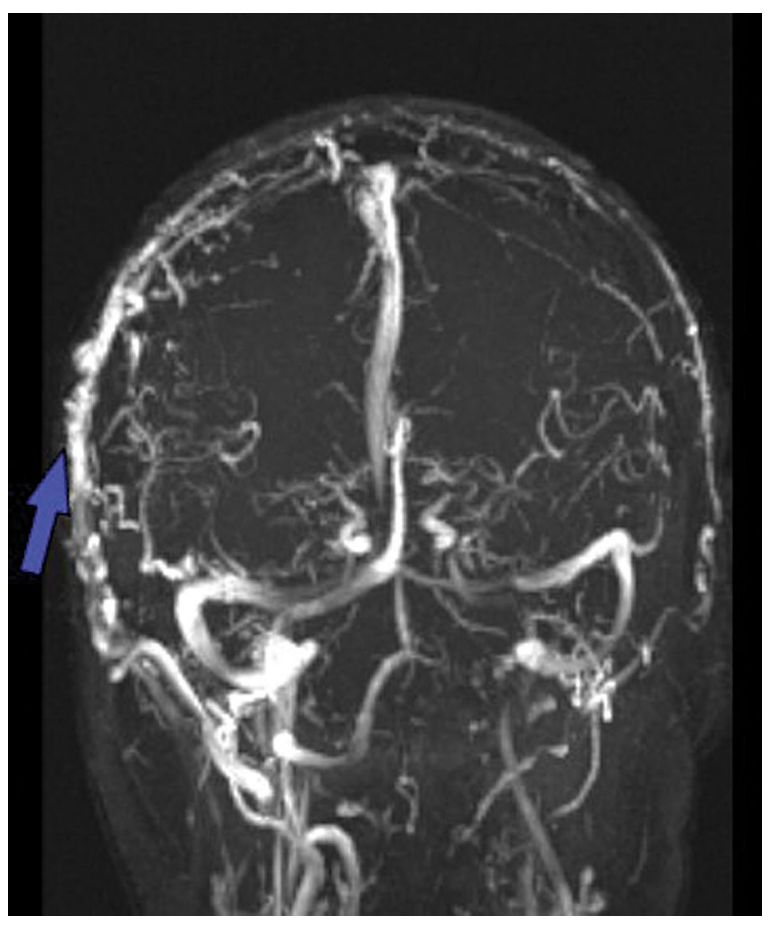

Figure 2. Magnetic resonance angiography showing wide, elongated, tortuous right temporal vessels (blue arrow).

gated superficial temporal vessels on the right side but a precise site of the connection between vessels was not identified (Fig. 2). Therefore CT angiography (CTA) imaging studies were performed. CTA revealed an AVM in the form of a fistula between the right superficial temporal artery and corresponding vein at the level of the right zygomatic arch (Figs. 3, 4). The fistula was situated just above the inferior margin of the right zygomatic arch and approximately $7 \mathrm{~mm}$ anteriorly to the anterior margin of the external acoustic meatus. The fistula had the form of a simple junction of walls between two adjacent vessels. The patient was referred to the Department of Vascu- 


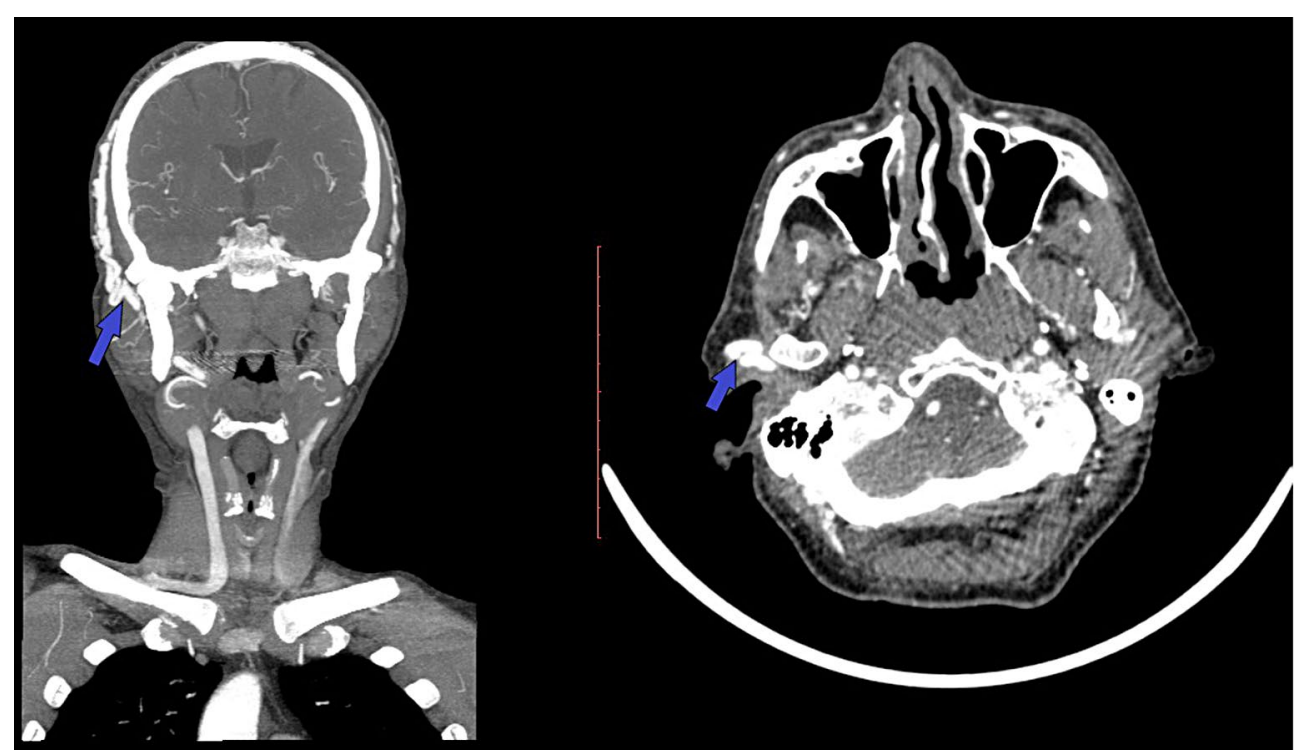

Figure 3. Blue arrow indicates fistula between the right superficial temporal artery and vein.

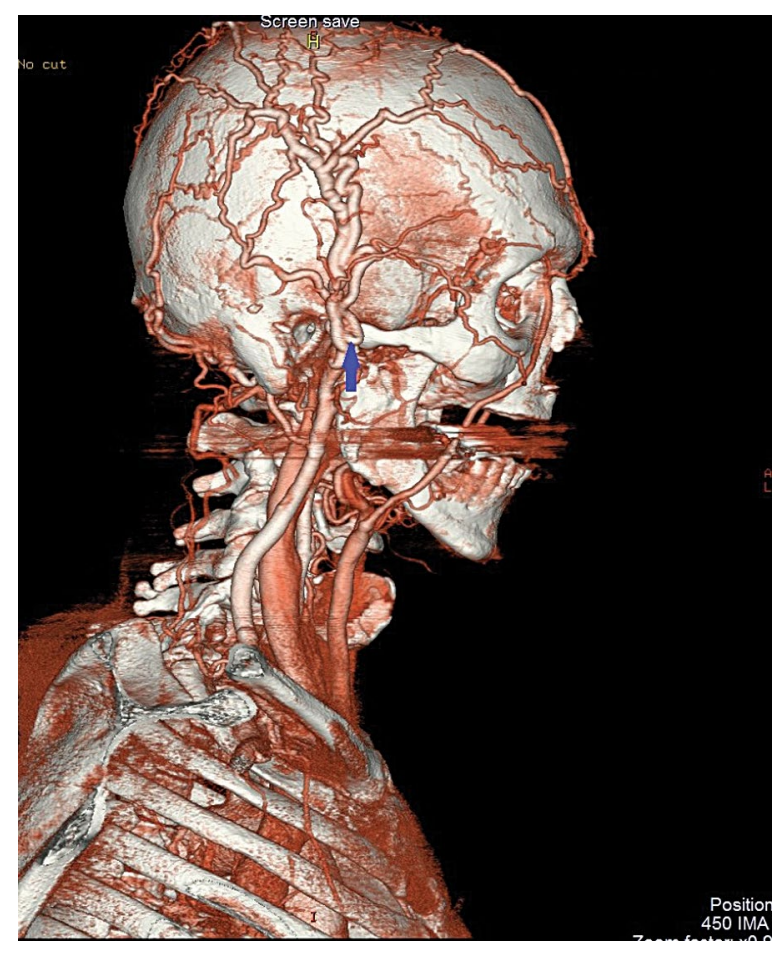

Figure 4. Blue arrow indicates the site of connection between the right superficial temporal artery and vein at the level of the zygomatic arch.

lar Surgery. The surgery was performed under local anaesthesia and consisted of ligation of the vessels forming the fistula that is superficial temporal artery and vein.

The patient reported the disappearance of the pulsatile tinnitus intra-operatively. One week fol- low-up exam revealed a superficial infection of the wound. Two week follow-up revealed proper wound healing with the initial infection being cured. During the post-operative period the patient had no other complications and was only complaining of minor pain in the post-surgical site. The follow-up Doppler ultrasound had shown normalisation of the flow profile in the right external carotid artery.

\section{DISCUSSION}

Headache is one of the most common complaints reported to primary care physicians. In this article we wanted to add a rather uncommon cause of headache. It is quite important to ask appropriate questions during the interview as well as to perform vigilant neurological examination in this group of patients. Arteriovenous fistulas of the scalp most often involve the superficial temporal artery as well as the occipital artery and their corresponding veins [1]. There are infrequent reports described in literature about AVFs between extracranial arteries and sinuses of the dura mater [8]. This configuration results in raised blood pressure in the affected sinus and internal jugular vein [6].

In summary, the most common symptoms of AVFs of the scalp are: localised headache, a pulsatile subcutaneous mass and pulsatile tinnitus in the ear. Due to decreased circulation within tissue, skin changes, like baldness or necrosis may also occur. The abnormalities found on physical examination are the following: bruit on auscultation of the mass, pulsatile vibration on palpation, and Terrier's sign. 
The fistulas can be divided into acquired and congenital according to the literature $[1,4,5]$. The former are traumatic (including iatrogenic trauma) in origin; however, sporadic nontraumatic cases are described $[8,10]$. Traumatic AVF is a rare condition [2]. It develops after accidental trauma of the head or after certain medical intervention (iatrogenic), specifically after temporomandibular joint surgery. Such fistulas may also develop as a complication of hair transplant surgery or neurosurgery $[3,5,9]$. The symptoms may develop immediately after the trauma or months to years following it. There is a hypothesis, that the onset of symptoms is strictly related to the pathomechanism of fistula formation. Namely this refers to the process of simultaneous arterial and venous wall laceration creating a single, direct connection between vessels. This process results in immediate patient complaints. The late clinical manifestation is related to the disruption of vasa vasorum. Their endothelium cells divide and form small vascular channels joining the adjacent vein. This process of formation may last up to a year, hence the delayed occurrence of symptomatology [2, 3].

Congenital fistulas may be present at birth or remain asymptomatic until adulthood [6]. Some factors are known to increase circulation within these malformations, which makes them symptomatic. These factors include: hormonal changes, pregnancy, trauma, vasomotor disturbance and inflammation [8]. There are several hypotheses explaining the formation of this pathology: the persistence of primitive arteriovenous channels and capillary agenesis, presence of vascular hamartomas (from which they may originate), and formation at sites of arteriovenous crossing [8].

In some cases it is difficult to establish the origin of the fistula. Patient may not be able to provide obvious history of head trauma. As our patient denied having trauma of the head, we can presume that his case was non-traumatic, but, due to history of alcohol addiction, traumatic aetiology cannot be excluded. In the situation of high suspicion of AVF the primary imaging study of choice is duplex-Doppler ultrasound of the carotid arteries. On the side of the pathology, the blood flow in the external carotid artery is low-resistant, turbulent, and accelerated [11]. However, the disadvantage of this examination is the inability to localise the site of the fistula. Therefore, the next step should be to perform a MRA with special attention to the area of patient complaint. According to liter- ature, it is more sensitive than CTA in searching for the site of pathologic arteriovenous leak. This stands in opposition to our case, in which CTA was the more precise examination [3]. Angiography (DSA) is the gold standard for diagnosis of AVF $[3,11]$.

The following ways of treatment are possible:

— ligation of feeding arteries and veins;

- total surgical excision;

- transarterial or transvenous embolisation and intralesional injection of sclerosant medication [3];

- combination of these methods [7].

The method of choice is surgical excision because it gives the lowest rate of recurrence. In patients with high risk of skin necrosis, ligation or embolisation are the preferred methods even though they carry a higher recurrence rate [3].

\section{CONCLUSIONS}

Arteriovenous fistula of the scalp vessels is a rare condition. Nevertheless, it should be considered in the differential diagnosis of localised headache with scalp pain.

\section{Acknowledgements}

We would like to express our gratitude to Pawel Nitkowski, MD, the vascular surgeon who operated the patient and to Leopold Bakon, MD, the radiologist, who diagnosed the above described pathology.

\section{REFERENCES}

1. Bret J, Kunc Z. Fistula between three main cerebral arteries and a large occipital vein . J Neurol Neurosurg Psychiatry. 1969: 32(4): 308-312, doi: 10.1136 jnnp.32.4.308, indexed in Pubmed: 5807870.

2. Júnior OC, Abreu M, Abreu G, et al. Traumatic arteriovenous fistula of the superficial temporal artery. J Vasc Brasil. 2014; 13(1): 39-42, doi: 10.1590/ jvb.2014.008.

3. Feng Li, Shugan Z, Yuguang L, et al. Traumatic arteriovenous fistula of the superficial temporal artery. J Clin Neurosci. 2007; 14(6): 595-600, doi: 10.1016/j. jocn.2006.04.011, indexed in Pubmed: 17379525.

4. Khodadad G. Arteriovenous malformations of the scalp. Ann Surg. 1973; 177(1): 79-85, doi: 10.1097/00000658-197301000-00015.

5. Ki HJ, Lee HK, Hur JW, et al. Post-Traumatic arteriovenous fistula of the scalp. J Korean Neurosurg Soc. 2015; 58(3): 298-300, doi: 10.3340/ jkns.2015.58.3.298, indexed in Pubmed: 26539278.

6. Komatsu Y, Narushima K, Kobayashi E, et al. Congenital arteriovenous malformation of the scalp--case report. Neurol Med Chir (Tokyo). 1989; 29(3): 230-234 doi: $10.2176 / \mathrm{nmc} 29.230$ indexed in Pubmed: 2477728.

7. Komiyama M, Nishikawa M, Kitano S, et al. Non-traumatic arteriovenous fistulas of the scalp treated by a combination of embolization and surgical removal. Neurol Med Chir (Tokyo). 1996; 36(3): 162-165, doi: 10.2176/ nmc.36.162, indexed in Pubmed: 8869152.

8. Mehmet $\mathrm{S}$, Alptekin $\mathrm{Y}$, Mustafa G, et al. Nontraumatic scalp arteriovenous fistula in an adult: technical report on an illustrative case. Surg Neurol. 2008; 70(2): 194-197, doi: 10.1016/j.surneu.2007.04.018, indexed in Pubmed: 18291475.

9. Miekisiak G, Mis M, Sandler A, et al. latrogenic arteriovenous fistula of the superficial temporal artery. Oral Maxillofac Surg. 2008; 12(4): 219-221, doi: 10.1007/s10006-008-0133-5, indexed in Pubmed: 18813963.

10. Morandi X, Godey B, Riffaud L, et al. Nontraumatic arteriovenous fistula of the superficial temporal artery. Otolaryngol Head Neck Surg. 2001; 124(5): 588-589, doi: 10.1067/mhn.2001.115088, indexed in Pubmed: 11337672.

11. Teng TY, Yun-Yu L, Ulf S, et al. Pulsatile tinnitus in a case of traumatic temporal extradural arteriovenous fistula: carotid duplex sonography findings before and after embolization. J Clin Ultrasound. 2008; 36(7): 432-436, doi: 10.1002/ jcu.20505, indexed in Pubmed: 18561342. 